10.1515/astro-2017-0285

Baltic Astronomy, vol.20, 211-220, 2011

\title{
GRIGORI KUZMIN AND STELLAR DYNAMICS
}

P. Tim de Zeeuw ${ }^{1,2}$ and Glenn van de $\mathrm{Ven}^{3}$

1 European Southern Observatory, Karl-Schwarzschild-Str. 2, 85748 Garching, Germany; tdezeeuw@eso.org

2 Sterrewacht Leiden, Leiden University, Postbus 9513, 2300 RA Leiden, The Netherlands

3 Max-Planck Institute for Astronomy, Königstuhl 17, 69117 Heidelberg, Germany; glenn@mpia.de

Received: 2011 July 09; accepted: 2011 July 21

\begin{abstract}
Grigori Kuzmin was a very gifted dynamicist and one of the towering figures in the distinguished history of the Tartu Observatory. He obtained a number of important results in relative isolation which were later rediscovered in the West. This work laid the foundation for further advances in the theory of stellar systems in dynamical equilibrium, thereby substantially increasing our understanding of galaxy dynamics.
\end{abstract}

Key words: stellar dynamics - galaxies: elliptical and lenticular, cD - galaxies: kinematics and dynamics - galaxies: structure

\section{INTRODUCTION}

At the scientific symposium celebrating the 200th anniversary of the Tartu Observatory, it is appropriate to consider the work of one of its leading astronomers, Grigori G. Kuzmin. Kuzmin was born in 1917, was a student at Tartu University from 1936 to 1940, and became an assistant at the Observatory in the same year. He received his $\mathrm{PhD}$ degree in 1952 and continued to work at the Observatory until after his retirement in 1982. He passed away in 1988, having made fundamental advances in the theory of galaxy dynamics.

In this contribution we concentrate on Kuzmin's work on galaxy models with separable potentials, from spherical to axisymmetric to triaxial (Sections 2-4). The stellar orbits in these galaxy models all have three exact integrals of motion which are quadratic functions of the velocity components, allowing the calculation of many properties by analytic means. The separable models not only provide insight into the internal orbital structure and formation of galaxies, but they are also essential for testing more sophisticated numerical modelling approaches (Section 5). Our conclusions follow in Section 6. 


\section{DYNAMICAL MODELS FOR GALAXIES}

In order to understand the dynamics of a collisionless stellar system such as the Milky Way, the goal is to find the phase-space distribution function $f(\vec{x}, \vec{v}) \geq 0$ which provides the distribution of stars over all positions $\vec{x}$ and all velocities $\vec{v}$. The mass-density distribution $\rho(\vec{x})$ follows by integrating $f(\vec{x}, \vec{v})$ over the velocities: $\rho=\int f \mathrm{~d}^{3} \vec{v}$. The velocities derive from the gravitational potential $\Phi(\vec{x})$ via Newton's equation of motion $\mathrm{d} \vec{v} / \mathrm{d} t=-\nabla \Phi(\vec{x})$. If the stellar system is self-consistent, then the density is related to the potential by Poisson's equation $4 \pi G \rho(\vec{x})=\nabla^{2} \Phi(\vec{x})$.

Traditionally two main approaches are taken to construct dynamical models. Either one chooses a form for $f$ in order to find $\rho$, which requires making a sensible Ansatz for $f$ based on some pre-conceived notion on the galaxy formation process, or one assumes $\rho$ and attempts to find $f$ by solving an integral equation. In both cases it is advantageous to use Jeans' (1915) theorem which states that $f$ depends on the six phase-space variables $\vec{x}$ and $\vec{v}$ only through the single-valued (isolating) integrals of motion admitted by the gravitational potential of the system. Since in non-spherical systems there are generally at most three such integrals, application of Jeans' theorem reduces the number of variables in the problem of constructing a dynamical model.

The second order velocity moments $\left\langle v_{i} v_{j}\right\rangle$ of $f$ (with $i, j=1,2,3$ ) are related directly to the density and the potential by the equations of stellar hydrodynamics, also called the Jeans equations. They can often be solved without knowledge of $f$, but this generally requires additional assumptions about the shape of the velocity distribution. Moreover, such Jeans models are not guaranteed to correspond to a distribution function $f$ that is everywhere non-negative, i.e., physical.

It is useful to consider spherical models. In this case the Hamilton-Jacobi equation separates in standard spherical coordinates $(r, \theta, \phi)$, and the motion of each star is governed by four isolating integrals of motion: the energy $E$, and the three components $L_{x}, L_{y}$ and $L_{z}$ of the angular momentum vector. All orbits are planar rosettes (with the maximum angular momentum circular orbit and the zero angular momentum radial orbit as extremes). The mass distribution is defined by specifying the density profile $\rho(r)$, and the associated gravitational potential $\Phi(r)$ can be found by carrying out two single weighted integrations of $\rho(r)$. There is only a single Jeans equation. Many self-consistent models have been constructed by analytic means. These include isotropic models with $f=f(E)$ found via an Abel inversion given the density $\rho(r)$ (Eddington 1916), circular orbit models in which only orbits with zero radial action are populated, the Osipkov (1979)/Merritt (1985) models in which $f=F\left(E+a L^{2}\right)$ and many more general distribution functions $f=f(E, L)$ with $L$ the total angular momentum.

Over the past century many papers were written on the construction of spherical models. Popular mass models are Hénon's (1959) isochrone and the $\gamma$-models (e.g., Dehnen $1993^{1}$ ), which include the Jaffe (1983) and Hernquist (1990) models. Many of these were, in fact, studied much earlier by Kuzmin and collaborators, in particular Veltmann and later Tenjes. They derived density profiles, Jeans solutions and distribution functions. The results were not well known in the Western literature, but were summarised in English in Kuzmin (1993). However, Kuzmin's main contribution lies elsewhere.

1 Also derived by M. Franx in unpublished notes dated 1988. 


\section{KUZMIN'S PIONEERING WORK}

When Kuzmin started working on galaxy dynamics in Tartu in the early nineteen forties, under very difficult circumstances, the field concentrated on the Milky Way, for which many basic properties were still poorly known. This included the mass distribution, the associated gravitational potential, the nature of the three-dimensional stellar orbits other than those provided by the epicyclic approximation for nearly-circular orbits, and the phase-space distribution function. He contributed to all these topics.

It was known from classical mechanics that in a flattened axisymmetric system such as the Milky Way, the energy $E$ and the angular momentum component $L_{z}$ parallel to the symmetry axis [in cylindrical coordinates $(R, \phi, z)]$, are integrals of motion. If the phase space distribution function $f$ would depend only on $E$ and $L_{z}$ (by the Jeans Theorem), then the second moments of the stellar motions observed, e.g., near the Sun, should obey the relations $\left\langle v_{R}^{2}\right\rangle=\left\langle v_{z}^{2}\right\rangle$ and $\left\langle v_{R} v_{z}\right\rangle=0$. But it was already clear from the observational data that $\left\langle v_{R}^{2}\right\rangle \neq\left\langle v_{z}^{2}\right\rangle$ and $\left\langle v_{R} v_{z}\right\rangle \neq 0$. This strongly suggested that the distribution function $f$ of the Milky Way depends on a third parameter, or in other words that the Galactic potential must support a third integral of motion $I_{3}$. The nature of this integral was not well understood, and at the time only an approximate expression been derived for nearly circular orbits with small vertical harmonic oscillations (Lindblad 1933).

Non-spherical gravitational potentials with three exact integrals of motion $E$, $I_{2}$ and $I_{3}$ had been known for half a century (Stäckel 1890, Eddington 1915, Clark 1937), but had received relatively little attention. The integrals $I_{2}$ and $I_{3}$ are quadratic functions of the velocity components. Chandrasekhar (1942) assumed that $f=f\left(E+a I_{2}+b I_{3}\right)$ for such systems (the Ellipsoidal Hypothesis), and attempted to derive the associated density $\rho$. He demonstrated that the only self-consistent models satisfying this assumed form for $f$ are spherical, and hence inconsistent with flattened systems such as the Milky Way. Interest in such spherical models was revived by Osipkov (1979) and Merritt (1985), but Chandrasekhar's result did not encourage further work on the flattened separable models. There was also little interest in pursuing the opposite route: to try to solve for $f$ for densities $\rho$ that have a separable potential. Van Albada (1952) (wrongly) demonstrated that oblate axisymmetric separable potentials are not associated with mass distributions that are everywhere non-negative, suggesting that their usefulness for stellar dynamics was severely limited.

In the mean time, Kuzmin had in fact made very significant progress in this area. He had worked on mass models for our Galaxy built by adding nonhomogeneous ellipsoids (work later repeated by e.g., Burbidge, Burbidge \& Prendergast 1959), had demonstrated that only isolating integrals of motion should be used in Jeans' Theorem (well before Lynden-Bell's 1962b paper on the subject), and, most significantly, had derived many interesting results for axisymmetric models with an exact third integral of motion. The results were collected in his seminal PhD Thesis (Kuzmin 1952 ${ }^{2}$ ), and many of these were published in the decade that followed.

Kuzmin considered axisymmetric mass models with a gravitational potential

$$
\Phi_{S}=\frac{F(\lambda)-F(\nu)}{\lambda-\nu}
$$

\footnotetext{
${ }^{2}$ Translated into English by Tenjes and Einasto, including additions from 1969.
} 
where $(\lambda, \phi, \nu)$ are prolate spheroidal coordinates, with $(\lambda, \nu)$ the two roots of

$$
\frac{R^{2}}{\tau+\alpha}+\frac{z^{2}}{\tau+\gamma}=1,
$$

and the constants $\alpha$ and $\gamma$ such that $-\gamma \leq \nu \leq-\alpha \leq \lambda$, and $F(\tau)$ an arbitrary smooth function $(\tau=\lambda, \nu)$. Surfaces of constant $\lambda$ are spheroids, and surfaces of constant $\nu$ are hyperboloids of two sheets, respectively. In these potentials, the Hamilton-Jacobi equation separates in $(\lambda, \phi, \nu)$, resulting in three exact integrals of motion $E, L_{z}$ and $I_{3}$, which are quadratic in the velocities and can be written explicitly. Kuzmin showed that such potentials have useful associated densities, given by a simple formula, and that the density $\rho(R, z) \geq 0$ if and only if $\rho(0, z) \geq$ 0, a result now known as Kuzmin's theorem (de Zeeuw 1985b).

Kuzmin assumed a simple form for the density profile along the short axis, $\rho(0, z)$, where $z^{2}=\tau+\gamma$, which in the notation used here would be equivalent to:

$$
\frac{d}{d \tau}(\tau+\gamma)^{1 / 2} F^{\prime}(\tau) \propto \tau^{-n / 2} .
$$

He showed that the case $n=3$ provides a fair approximation to the Milky Way potential as it was known at the time (without dark halo); the model is nearly spheroidal and is in fact a flattened generalisation of Hénon's isochrone. The density (and potential) can be written explicitly in terms of $R$ and $z$, avoiding the need to use spheroidal coordinates. The case $n=4$ corresponds to an exactly spheroidal model $\rho(R, z)=\rho_{0} /\left(1+m^{2}\right)^{2}$ with $m^{2}=R^{2} / a^{2}+z^{2} / c^{2}$ and $\alpha=$ $-a^{2}, \gamma=-c^{2}$. In the limit of extreme flattening both the $n=3$ and the $n=4$ models reduce to an infinitesimally thin disk with surface density $\Sigma(R)=\Sigma_{0} /(1+$ $\left.R^{2} / a^{2}\right)^{3 / 2}$. This is the Kuzmin (1953) disk which was rediscovered by Toomre (1963). It has many remarkable properties, including the fact that the potential above the disk is equal to that of a point mass at $(R=0, z=-a)$ below the disk and the potential below the disk is that of a point mass at $(R=0, z=+a)$ above the disk. Kuzmin furthermore noted that the model with $n=n_{0}$ is the weighted sum of models with $n>n_{0}$; this built on his pioneering 1943 work on construction of models by superposition of inhomogeneous spheroids.

The stellar orbits in oblate separable models are bounded by the orthogonal confocal coordinate surfaces, and are now called short-axis tubes. Their properties are similar to those of the orbits in the Milky Way found numerically by Ollongren (1962) using Schmidt's (1956) mass model. Kuzmin \& Kutuzov (1962) calculated the $f\left(E, L_{z}\right)$ for the model $n=3$. They realised that $\rho(R, z)$ can be written explicitly as $\rho(R, \Phi)$ without any reference to spheroidal coordinates, which allows computing $f\left(E, L_{z}\right)$ via series expansions à la Fricke (1952). This work occurred in parallel with Lynden-Bell's (1962a) construction of self-consistent flattened Plummer models using integral transforms. But Kuzmin \& Kutuzov apparently did not investigate three-integral distribution functions for oblate (and the related prolate) density distributions. This was left to Dejonghe \& de Zeeuw (1988) who made full use of the many elegant properties of the Kuzmin-Kutuzov mass model, derived a closed form for $f\left(E, L_{z}\right)$ (see also Batsleer \& Dejonghe 1993) as well as a number of three-integral distribution functions, and computed the projected surface density and the observed kinematic properties. 


\section{TRIAXIAL GALAXY MODELS}

Kuzmin also generalised much of his work on oblate separable models to those with triaxial shapes. This work is largely unpublished except for a very concise four-page summary (Kuzmin 1973) in the proceedings of the Alma Ata conference in 1972, which was translated into English in Kuzmin (1987). The paper considers triaxial models with potentials separable in confocal ellipsoidal coordinates $(\lambda, \mu, \nu)$ defined as the three roots for $\tau$ of

$$
\frac{x^{2}}{\tau+\alpha}+\frac{y^{2}}{\tau+\beta}+\frac{z^{2}}{\tau+\gamma}=1,
$$

with $(x, y, z)$ the usual Cartesian coordinates, and with constants $\alpha, \beta, \gamma$ such that $-\gamma \leq \nu \leq-\beta \leq \mu \leq-\alpha \leq \lambda$. For potentials of the form

$$
\Phi_{S}=\frac{F(\lambda)}{(\lambda-\mu)(\lambda-\nu)}+\frac{F(\mu)}{(\mu-\nu)(\mu-\lambda)}+\frac{F(\nu)}{(\nu-\lambda)(\nu-\mu)},
$$

all orbits have three exact integrals of motion $E, I_{2}$ and $I_{3}$, quadratic in the velocities. Kuzmin noted that the orbits can be divided into four main families, that the density associated to $\Phi_{S}$ is non-negative, i.e., $\rho(x, y, z) \geq 0$ if and only if $\rho(0,0, z) \geq 0$, and that it can be expressed elegantly via a 'Kuzmin formula'. The set of models includes an exactly ellipsoidal model, also known as the 'perfect ellipsoid':

$$
\rho(x, y, z)=\rho_{0} /\left(1+m^{2}\right)^{2}, \quad \text { with } \quad m^{2}=x^{2} / a^{2}+y^{2} / b^{2}+z^{2} / c^{2},
$$

where $\gamma=-c^{2}, \beta=-b^{2}$ and $\alpha=-a^{2}$. The four major orbit families are referred to as box orbits, inner long-axis tube orbits, outer long-axis tube orbits, and short-axis tube orbits, and are identical to the four major orbit families found in Schwarzschild's (1979) numerical model for a stationary triaxial galaxy with a constant density core (de Zeeuw 1985a).

All these results for triaxial models were obtained via an independent route by the first author in the period 1982-1985, following a suggestion by Lynden-Bell to 'look into how well separable potentials could be made to fit a general potential in the finite density core of a galaxy' (de Zeeuw \& Lynden-Bell 1985). This lead to the discovery of the perfect ellipsoid (Equation 6) in a fairly roundabout way - as the ellipsoidal model for which the series expansion of the potential fits a separable one to all orders - and to the subsequent rediscovery of the Kuzmin (1956) paper on oblate separable models. The latter inspired the independent derivation of the Kuzmin formula for the triaxial case, and was followed by the full derivation of the gravitational potential for oblate, prolate and triaxial models given as the sum of two weighted single integrations of the density profile along the short axis (de Zeeuw 1985b; de Zeeuw, Peletier \& Franx 1986). It was Jaan Einasto who cheerfully pointed out to the first author in 1985 during the Princeton IAU Symposium on Dark Matter that 'Kuzmin had already done it all for triaxial models many years earlier'. This overstated the case a little, as the derivation of the potential given the short-axis density profile was genuinely new. But the trail led to the Alma Ata conference contribution already mentioned, and the decision to have it translated into English and included as an Appendix of the proceedings of IAU Symposium 127 (Kuzmin 1987). The text was carefully checked by Kuzmin himself about one year before he passed away. 
As Kuzmin knew, each separable triaxial model can be written as the weighted integral of constituent perfect ellipsoids, with the weight function found by means of a Stieltjes transform. It follows that the projected surface density is the same weighted integral of the projections of the constituent ellipsoids, which are elliptic disks. This property led to a new and powerful method to find the threedimensional gravitational potential of non-axisymmetric disks (Evans \& de Zeeuw 1992). Franx (1988) had already deduced general properties of the projections of the separable models. All these properties are in fact shared by a much larger set of models: each ellipsoid $\rho=\rho_{0} /\left(1+m^{2}\right)^{p}$ with $p=n$ or $p=n / 2$ generates a similar family (de Zeeuw \& Pfenniger 1988; Evans \& de Zeeuw 1992), but the motion is separable only for the models generated by the $p=2$ ellipsoids.

The second moment tensor is diagonal for the separable models, and the diagonal elements are related to $\rho$ and $\Phi_{S}$ by the Jeans equations, which are three partial differential equations for three unknowns (Lynden-Bell 1960). Kuzmin and others had derived special solutions for the axisymmetric and elliptic disk limiting cases, but the general equations for triaxial geometry resisted solution for more than forty years, until van de Ven et al. (2003) found it by classical methods. As for all Jeans solutions, they are not guaranteed to correspond to a physical model with $f \geq 0$, but do allow efficient calculation of the second velocity moments.

It has also been possible to construct analytic self-consistent models, but the existence of more than one major orbit family, in fact four, implies that $f\left(E, I_{2}, I_{3}\right)$ is not uniquely defined by $\rho(\lambda, \mu, \nu)$, and certain assumptions have to be made. Populating only the tube orbits with zero radial action (thin tubes) together with the box orbits, Hunter \& de Zeeuw (1992) constructed models that can be considered as the triaxial generalisation of the circular orbit spheres. Van de Ven et al. (2008) gave a comprehensive description of the so-called Abel models in which $f=\sum_{i} f_{i}\left(E+a_{i} I_{2}+b_{i} I_{3}\right)$, with $a_{i}$ and $b_{i}$ constants for each component $i$. These had been studied earlier by Dejonghe \& Laurent (1991) and Mathieu \& Dejonghe (1999), and are triaxial generalisations of the spherical Osipkov-Merritt models.

The above brief summary shows that through Kuzmin's work and the subsequent follow-up, the theory of stationary triaxial dynamical models is now as comprehensive as that for spheres. This also means that by matching these models with observations of galaxies - such as their observed surface brightness to infer the intrinsic density, and their observed stellar kinematics to constrain the gravitational potential and internal velocity distribution - we can gain insight in the internal dynamics of a much larger range of non-spherical stellar systems, including early-type galaxies.

\section{EARLY-TYPE GALAXIES}

Measurements of the stellar kinematics of early-type galaxies with integral-field spectrographs such as SAURON (Bacon et al. 2001) show that they can be divided into slow rotators and fast rotators, different from their morphological classification in ellipticals and lenticulars (Emsellem et al. 2007, 2011). Most fast rotators, including lenticular as well as many elliptical galaxies, seem to be close to oblate axisymmetric disk-like systems (Cappellari et al. 2007). On the other hand, various slow-rotator elliptical galaxies show clear deviations from axisymmetry in their photometry such as isophotal twists, as well as in their kinematics such as kinematic misalignment and kinematically decoupled components (see e.g. Emsellem et al. 2004, and Figure 1), indicating that they are triaxial. 


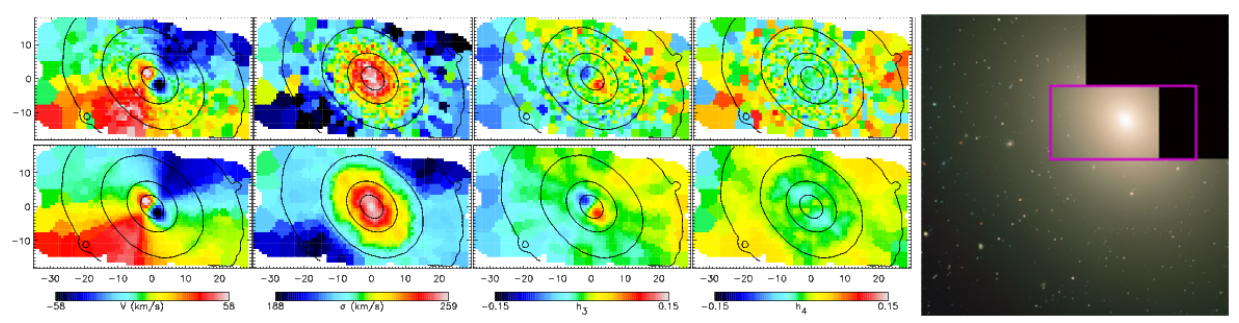

Fig. 1. Triaxial dynamical model of the elliptical galaxy NGC 4365. Left: Twodimensional stellar kinematic observations of NGC 4365 obtained with the integral-field spectrograph SAURON (top row) accurately fitted with our numerical implementation of Schwarzschild's orbit superposition method in triaxial geometry (bottom row). From left to right: mean line-of-sight velocity and dispersion, and higher-order Gauss-Hermite velocity moments, which quantify deviations from a Gaussian velocity profile. Right: The black contours drawn on the maps are the isophotes derived from Hubble Space Telescope imaging of NGC 4365 shown on the right-hand side with a magenta box indicating the extent of the SAURON observations. Whereas the imaging shows a smooth elliptical luminosity distribution, the kinematics reveal a complex non-axisymmetric orbital distribution with an (apparent) kinematically decoupled core.

The separable triaxial galaxy models described above are able to capture much of the rich internal dynamics of these slow-rotator galaxies. However, the presence of central density cups and super-massive black holes imply that there are no global integrals $I_{2}$ and $I_{3}$. The tube orbits are relatively unaffected, but the box orbits are replaced by a mix of so-called boxlets (higher-order resonant orbits) and chaotic orbits (e.g., Gerhard \& Binney 1985). While separable models are very useful to provide insights as well as tests (e.g., van de Ven et al. 2008), to model these elliptical galaxies in detail, a more general technique such as Schwarzschild's (1979) numerical orbit superposition method is needed.

Schwarzschild's method allows for an arbitrary gravitational potential, with possible contributions from a central black hole and a dark matter halo. The equations of motions are integrated numerically for a representative library of orbits. Next, the orbital weights are determined for which the combined density and higher order velocity moments best fit simultaneously the observed surface brightness and (two-dimensional) kinematics. The resulting distribution of (positive) orbital weights represents the distribution function (Vandervoort 1984), which is, by construction, guaranteed to be non-negative everywhere. Our recent implementation in triaxial geometry (van de Ven et al. 2008, van den Bosch et al. 2008, van den Bosch \& van de Ven 2009), allows the construction of accurate models for slow-rotator galaxies and to establish their internal phase-space structure.

One of these galaxies is NGC 4365, a giant E3 galaxy with a core that appears to rotate around the short axis (Surma \& Bender 1995; Davies et al. 2001), almost perpendicular $\left(\sim 82^{\circ}\right)$ to the main body rotation around the long-axis (Figure 1). The customary interpretation has been that this core is kinematically distinct (a so-called KDC) and a remnant of a last major accretion $\sim 12$ Gyr ago. However, triaxial Schwarzschild models fitted to the SAURON stellar kinematics (van den Bosch et al. 2008; Figure 1), revealed that the KDC is part of the single triaxial structure: short axis tube orbits dominate throughout, but half of them counterrotate leading to vanishing net rotation around the short-axis, except in the centre due to a small in-balance leading to the appearance of a KDC. The intrinsic shape 
changes from oblate triaxial in the central regions to prolate triaxial in the outer parts where long-axis tube orbits provide the net rotation seen in the observed velocity field.

Triaxial Schwarzschild models for another dozen of these slow-rotator ellipticals reveal a similar picture of galaxies dominated by short axis tube orbits with cancelling rotation apart from a small in-balance in the centre leading to the KDCs. In addition, these slow-rotator galaxies contain on average $20 \%$ box orbits consistent with their mildly triaxial shape (van den Bosch et al., in prep.). Several fastrotator galaxies also contain KDCs, but typically a magnitude smaller in size, and they seem to be truly decoupled axisymmetric disk-like components with a younger stellar population (see also McDermid et al. 2006). The slow-rotator galaxies with these apparent KDC's, in harmony with their smooth age and metallicity gradients, provide a true challenge for (cosmological) merger simulations: Dry mergers over-predict the fraction of box orbits (e.g., Jesseit et al. 2005) but invoking gas seems to require special conditions to make the merger remnants as round as the observed slow-rotator ellipticals (e.g., Hoffman et al. 2010; Bois et al. 2011).

\section{CONCLUDING REMARKS}

Kuzmin also contributed to other areas of galactic dynamics, and trained a number of students, amongst which Einasto, Kutuzov and Veltmann. The relative isolation in which he worked resulted in a delayed recognition of many of his achievements in the West. This was due in part to limited possibilities for publishing, information exchange and travel in the Soviet era, and in part to provincialism, as few in the West were able to read Russian. Kuzmin's seminal 1956 paper was published in the Russian Astronomical Journal in the last year before it started to be translated in English. His papers often had a short English summary attached, and he sent English synopses of his work to key astronomers, amongst which Lynden-Bell, Oort and others, but these papers were not widely distributed. Perek's (1962) review did help advertise his results, but even so, some of his work was independently rediscovered. Today's on-line availability of preprints and journals, including new and old Baltic Astronomy publications, and the strongly improved possibilities for travel, should go a long way towards avoiding that genuine discoveries are overlooked and repeated.

A number of the ideas in Kuzmin's (1956) paper on oblate separable models were generalised to prolate and triaxial models and in turn led to further developments, as described in Sections 3 and 4. These contributed significantly to bringing the modelling of separable triaxial systems to the level reached for spherical systems: a theory of mass models and their projections, full understanding of the orbits, general solution of the Jeans equations, and construction of anisotropic distribution functions. This effort is valuable for testing more sophisticated numerical triaxial models and for gaining insight into the dynamical structure of early-type galaxies. As such, Kuzmin's work contributed substantially to our understanding of galaxy dynamics. He belongs in the list of very famous astronomers associated with Tartu Observatory.

ACKNOWLEDGMENTS. The authors are grateful to Jaan Einasto and Peeter Tenjes for helpful discussions and for a preview of the English translation of Kuzmin's PhD thesis and subsequent addenda, and to Anu Reinart and Laurits Leedjärv for generous hospitality at the Tartu Observatory. 


\section{REFERENCES}

Bacon R. et al. 2001, MNRAS, 326, 23

Batsleer P., Dejonghe H. 1993, A\&A, 271, 104

Bois M. et al. 2011, MNRAS, in press (arXiv:1105.4076)

Burbidge E. M., Burbidge G. R, Prendergast K. H. 1959, ApJ, 130, 739

Cappellari M. et al. 2007, MNRAS, 379, 418

Chandrasekhar S. 1942, Principles of Stellar Dynamics, Univ. of Chicago Press

Clark G. L. 1937, MNRAS, 97, 182

Davies R. L. et al. 2001, ApJ, 548, L33

Dehnen W. 1993, MNRAS, 265, 250

Dejonghe H., de Zeeuw P. T. 1988, ApJ, 333, 90

Dejonghe H., Laurent D. 1991, MNRAS, 252, 606

de Zeeuw P. T. 1985a, MNRAS, 216, 273

de Zeeuw P. T. 1985b, MNRAS, 216, 599

de Zeeuw P. T., Lynden-Bell D. 1985, MNRAS, 215, 713

de Zeeuw P. T., Peletier R.F., Franx M. 1986, MNRAS 221, 1001

de Zeeuw P. T., Pfenniger D. 1988, MNRAS, 235, 949

Eddington A. S. 1915, MNRAS, 76, 37

Eddington A. S. 1916, MNRAS, 76, 572

Emsellem E. et al. 2004, MNRAS, 352, 721

Emsellem E. et al. 2007, MNRAS, 379, 401

Emsellem E. et al. 2011, MNRAS, 414, 888

Evans N. W., de Zeeuw P. T. 1992, MNRAS, 257, 152

Franx M. 1988, MNRAS, 231, 285

Fricke W. 1952, AN, 280, 193

Gerhard O. E., Binney J. J. 1985, MNRAS, 216, 467

Hernquist L. 1990, ApJ, 356, 359

Hénon M. 1959, AnAp, 22, 126

Hoffman L., Cox T. J., Dutta S., Hernquist L. 2010, ApJ, 723, 818

Hunter C., de Zeeuw P. T. 1992, ApJ, 389, 79

Jaffe W. 1983, MNRAS, 202, 995

Jeans J. H. 1915, MNRAS, 76, 70

Jesseit R., Naab T., Burkert A. 2005, MNRAS, 360, 1185

Kuzmin G. G. 1952, PhD Thesis, Tartu University

Kuzmin G. G. 1953, Tartu Astron. Obs. Teated, 1

Kuzmin G. G. 1956, Astr. Zh., 33, 27

Kuzmin G. G. 1973, Proc. All-Union Conf. Dynamics of Galaxies and Clusters, Alma Ata, ed. T.B. Omarov, p. 71

Kuzmin G. G. 1987, in Structure and Dynamics of Elliptical Galaxies, IAU Symp. No. 127, Reidel, p. 553

Kuzmin G. G., Veltmann U.-I.,K. 1993, in Galactic Bulges, IAU Symp. No. 153, Kluwer, p. 363

Kuzmin G. G., Kutuzov S. A. 1962, Bull. Abastumani Obs., 27, 82

Lindblad B. 1933, Handbuch der Astrophysik, Springer Verlag, 2, 1033

Lynden-Bell D. 1960, PhD Thesis, Cambridge University

Lynden-Bell D. 1962a, MNRAS, 123, 447 
Lynden-Bell D. 1962b, MNRAS, 124, 1

Mathieu A., Dejonghe H. 1999, MNRAS, 303, 455

McDermid R. M. et al. 2006, MNRAS, 373, 906

Merritt D. R. 1985, AJ, 90, 1027

Ollongren A. 1962, BAN, 16, 241

Osipkov L. 1979, Sov. Astron. Letters, 5, 42

Perek L. 1962, Advances in Astronomy and Astrophysics, 1, 165

Schmidt M. 1956, BAN, 13, 15

Schwarzschild M. 1979, ApJ, 232, 236

Stäckel P. 1890, Math. Ann., 35, 91

Surma P., Bender R. 1995, A\&A, 298, 405

Toomre A. 1963, ApJ, 138, 385

van Albada G. B. 1952, Proc. Royal Nederl. Acad. of Arts and Sciences, 55, No. 5,620

van de Ven G., Hunter C., Verolme E. K., de Zeeuw P. T. 2003, MNRAS, 342, 1056

van de Ven G., de Zeeuw P. T., van den Bosch R. C. E. 2008, MNRAS, 385, 614 van den Bosch R. C. E., van de Ven G., Verolme E. K., Cappellari M., de Zeeuw P. T. 2008, MNRAS, 385, 647

van den Bosch R. C. E., van de Ven G. 2009, MNRAS, 398, 1117

Vandervoort P. O. 1984, ApJ, 287, 475 